\title{
VARIAZIONI DELLE COSTANTI ARMONICHE DELLE MAREE COL LIVELLO DEL MARE
}

\author{
Silvio Polli
}

Premesse. - L'oscillazione della marea può essere considerata quale somma di oscillazioni elementari sinusoidali. Le più importanti di queste sono la componente lunare semidiurna principale M2, la solare semidiurna principale S2, la lunisolare declinazionale diurna $\mathrm{Kl}$, la lunare diurna principale $\mathrm{Ol}$, la solare diurna principale Pl e la lunare semidiurna ellittica maggiore N2. Per caratterizzare la marea in un dato posto si determinano le costunti armoniche delle maree componenti. Esse sono i valori delle semiampiezze $H$ (in $\mathbf{c m}$ ) delle singole onde componenti e le corrispondenti situazioni vere o assolute $\%$ (in gradi), che rappresentano il ritardo dell'alta marea rispetto al passaggio al meridiano di Greenwich dell'astro che produce quella marea. Invece della situazione vera $\%$ si usa, per maggior comodità, la situazione adattuta $g$, ciò̀ riferita al meridiano sul quale è regolata l'ora locale.

La marea si sviluppa in ciascun mare e in ogni suo punto con caratteri diversi che dipendono dalla forma del bacino che lo contiene. I valori delle costanti armoniche delle costituenti la marea mentre risultano diversi da luogo a luogo si mantengono, per uno stesso posto, costanti col tempo. Vi ì una stretta ma complessa dipendenza tra il valore delle costanti armoniche delle maree in un punto del bacino e la forma dello stesso. Una variazione anche minima di questo si riflette immediatamente sui valori di quelle. Inversamente, una variazione delle costanti armoniche denota un'alterazione nello spazio acqueo influente su quel punto. Tutto ciò supponendo costanti le cause astronomiche che yenerano le maree.

La forma spaziale di un mare varia col tempo soprattutto in quanto varia il suo livello medio. Questo può subire aumenti o abbassamenti che da un anno all'altro possono essere di qualche $\mathbf{c m}$.

In questa nota si metterà in evidenza come variazioni di pochi cm nell'altezza del livello medio dei mari Adriatico, Tirreno e Ionio comportino corrispondenti variazioni nei valori delle costanti armo- 
niche delle maree. La correlazione i particolarmente notevole per i valori del ritardo di fase. Ad un livello marino più alto corrisponde un magriore ritardo di fase, aumentano ciò $\mathrm{i}$ valori delle situazioni. L'influenza sulle ampiezze, per la piccola variazione della massa acquea, risulta invece meno apprezzabile.

Materiale utilizzato. - Le costanti armoniche delle marec di una località si calcolano applicando l'analisi armonica alla curva di marea registrata da un mareografo. Il lavoro d'analisi ì molto lungo e, dato che la variazione delle costanti is minima col tempo, viene eseguito per ciascun porto una volta sola.

Per l'Adriatico solamente Trieste dispone di più serie di analisi annue delle marec; tutte le altre stazioni hamno al masimo analisi di un anno. Per le coste italiane del Tirreno solamente Imperia, Civitavecchia, Napoli e Palermo dispongono ciascuna di più di una analisi annua. Dello Ionio ì stato possibile prendere in considerazione la sola stazione di Catania. L'esame pertanto si limiterà a queste 6 stazioni. Dato però il carattere e la evidenza della relazione, questi casi risultano sufficienti a mettere in rilievo non solo il fenomeno di dipendenza ma anche il suo ordine di grandezza.

A Trieste lo strumento reqistratore ì un mareografo Seilst-Fuess, con un rapporto di riduzione 1:10 ed uno scorrimento del forrlio di $30 \mathrm{~mm}$ per ora. E sistemato all'estremità nord del molo Sartorio, nell'interno del porto di Trieste. Le sue coordinate yeowrafiche sono: lat. $45^{\circ} 38^{\prime} 50^{\prime \prime} .5 \mathrm{~N}$ : long. $13^{\circ}+5^{\prime} 30^{\prime \prime} .5 \mathrm{E}$ Grcenwich. Sono state analizzate le registrazioni mareografiche degli anni completi 1905, 1911, 1921, 1931, 1941 e 1951.

A Imperia il mareografo è del tipo Uifficio Idrografico del Magistrato alle Acque di Venezia. Ha un rapporto di riduzione 1:5 ed uno scorrimento del forlio di $15 \mathrm{~mm}$ per ora. E situato nell'interno del Porto Maurizio presso la Capitaneria. Le sue coordinate geografiche sono: lat. $43^{\circ} 52^{\prime}, 7 \mathrm{~N}$ : long̣. $08^{\prime \prime} 0 \mathrm{I}^{\prime}, 4 \mathrm{E}$ Greenwich. Le analisi sono state dedotte dai due periodi di luglio 195]-marzo 1952 e aprile 1952-gennaio 1953.

A Civitarecchia il mareografo, del tipo come il precedente, $\dot{e}$ sistemato nell'interno del porto, nella Darsena Romana. Le sue coordinate geografiche sono: lat. $42^{\prime \prime} 05^{\prime} \mathrm{N}$; long. 11" $48^{\prime} \mathrm{E}$ Greenwich. Le tre serie di costanti armoniche sono state dedotte dai periodi: griugnodicembre 1951; gennaio-dicembre 1952: grennaio-agosto 1953. 
Napoli ha il mareografo, di tipo e caratteristiche come i due precedenti, sistemato nel porto nell'interno del molo a martello. Le sue coordinate sono: lat. $40^{\circ} 50^{\prime} \mathrm{N}$; long. $14^{\prime \prime} 16^{\prime}$ E Greenwich. I due periodi considerati comprendono gli anni 1951 e 1952 completi.

A Palermo, lo stesso modello di mareografo è situato nell'interno del porto, al lato ovest del Molo Nord. Le sue coordinate sono: lat. $38^{\prime \prime} 08^{\prime}, 0 \mathrm{~N}$; long. $13^{\prime \prime} 22^{\prime}, 5 \mathrm{E}$ Greenwich. Le tre serie di analisi armoniche comprendono i periodi febbraio-dicembre 1951; gennaio-aprilc 1952 e marzo-luglio 1953.

A Catania il mareografo è di tipo settimanale, con un rapporto di riduzione 1:5 e uno scorrimento del foglio di $72 \mathrm{~mm}$ per 24 ore. É situato nell'interno del porto al lato ovest del molo sporgente centrale. I.e sue coordinate sono: lat. $37^{\circ} 29^{\prime}, 9 \mathbb{N}$; long. $15^{\circ} 05^{\prime}, 8$ E Greenwich. I due periodi analizzati comprendono i due anni completi 1950 e 1951 .

Procedimento. - Sono stati utilizzati i mareogrammi dei periodi sopra indicati in quanto essi erano gli unici regolari e attualmente disponibili presso il Centro di Studi Talassografici.

Per il calcolo delle costanti armoniche si è adottato il metorlo elaborato da A. T. Doodson per successioni continue di valori orari estesi a 696 ore 29 giornit. Il calcolo separato per periorli di 29 giorni è necessario quando non si disponga di serie semestrali o annue di mareogrammi. Esso è pure conveniente per la possibilità di controllare la omogeneità e la regolarita della serie esaminata e delle corrispondenti costanti armoniche. Infatti, scostamenti forti nei valori successivi delle singole costanti indicano o errori di calcolo o irregolarita nel funzionamento dello strumento. Il tempo adottato è quello medio dell'Europa Centrale (T.M.E.C.). La situazione adottata $g$ è riferita allo stesso meridiano.

I livelli medi marini corrispondono agli stessi intervalli delle analisi e risultano dedotti quale media di tutte le altezze orarie comprese nei periodi stessi.

I valori indicati nelle tabelle aumentano verso l'alto e sono riferiti a determinati piani hoali collegati con le piastrine dei mareografi. Non è stato possibile riferirli alla rete altimetrica nazionale per mancanza dei dati di collegamento con i caposaldi della stessa.

La tal. 1 presenta, per le stazioni mareografiche considerate, le situazioni adattate $g$ in gradi sessagesimali per le componenti più importanti della marea e $i$ corrispondenti livelli medi. Per ciascun anno 
indicato si è pure dato il numero dei mesi analizzati. Le altezze dei livelli medi sono misurate in cm. La tall. 2 dà invece $i$ valori delle semiampiezze $H$ delle stesse componenti e i relativi livelli medi.

TAB. 1. - COSTANTI ARMONICHE DELLE MAREE E LIVELLI MEDI VALORI DELLA SITUAZIONE ADATTATA $g$ (in gradi)

\begin{tabular}{|c|c|c|c|c|c|c|c|c|c|}
\hline STAZIONE & Anno & Mesi & M2 & S2 & N2 & K1 & $\mathrm{Ol}$ & PI & L. M. \\
\hline \multirow[t]{6}{*}{ Trieste } & 1905 & 12 & 275 & 283 & 274 & 70 & 59 & 70) & 51,7 \\
\hline & 1911 & 12 & 275 & 282 & 271 & 69 & 60) & 69 & 50,0 \\
\hline & 1921 & 12 & 273 & 281 & 270 & 68 & 62 & 68 & 48,0 \\
\hline & 1931 & 12 & 276 & 284 & 280 & 72 & 63 & 72 & 57,8 \\
\hline & 1941 & 12 & 277 & 286 & 281 & 70 & 68 & 70 & 61,4 \\
\hline & 1951 & 12 & 278 & 286 & 275 & 71 & 62 & 71 & 64,8 \\
\hline \multirow[t]{2}{*}{ Imperia } & 1951 & 9 & 250 & 268 & 239 & 198 & 115 & 198 & 88,9 \\
\hline & 1952 & 10 & 251 & 269 & 244 & 196 & 120 & 196 & 90,7 \\
\hline \multirow[t]{3}{*}{ Civitavecchis } & 1951 & 6 & 258 & 281 & 245 & 202 & 120 & 202 & 80,0 \\
\hline & 1952 & 12 & 259 & 279 & 248 & 201 & 118 & 201 & 80,8 \\
\hline & 1953 & 6 & 258 & 281 & 244 & 203 & 106 & 203 & 78,9 \\
\hline \multirow[t]{2}{*}{ Napoli } & 1951 & 12 & 268 & 288 & 251 & 217 & 129 & 217 & 27,9 \\
\hline & 1952 & 12 & 269 & 289 & 254 & 217 & 135 & 217 & 30,2 \\
\hline \multirow[t]{3}{*}{ Palermo } & 1951 & 9 & 262 & 286 & 246 & 209 & 127 & 209 & 17,1 \\
\hline & 1952 & 4 & 262 & 284 & 249 & 211 & 122 & 211 & 14,6 \\
\hline & 1953 & 4 & 258 & 283 & 243 & 20) 2 & 116 & 202 & 7,8 \\
\hline \multirow[t]{2}{*}{ Catania } & 1951 & 12 & 87 & 95 & 95 & 58 & 52 & 58 & 33,5 \\
\hline & 1952 & 12 & 84 & 90 & 86 & 58 & 51 & 58 & 28,6 \\
\hline
\end{tabular}

TAB. 2. - COSTANTI ARMONICHE DELLE MAREE E LIVELLI MEDI VALORI DELLA SEMIAMPIEZZA $\mathrm{H}$ (in $\mathrm{cm}$ )

\begin{tabular}{|l|r|r|r|r|r|r|r|r|r|}
\hline \multicolumn{1}{|c|}{ STAZIONE } & Anno & Mesi & M2 & S2 & N2 & K1 & O1 & PI & L. M. \\
\cline { 2 - 6 } Trieste & 1905 & 12 & 26,5 & 16,0 & 4,5 & 18,8 & 6,0 & 6,2 & 51,7 \\
& 1911 & 12 & 26,3 & 16,3 & 4,8 & 18,3 & 5,2 & 6,0 & 50,0 \\
& 1921 & 12 & 26,2 & 15,7 & 4,7 & 18,5 & 5,5 & 6,1 & 48,0 \\
& 1931 & 12 & 26,9 & 15,9 & 4,6 & 18,9 & 5,2 & 6,2 & 57,8 \\
& 1941 & 12 & 26,9 & 15,9 & 4,5 & 19,0 & 5,7 & 6,4 & 6,4 \\
Imperia & 1951 & 12 & 26,9 & 16,3 & 4,5 & 18,2 & 5,8 & 6,0 & 64,8 \\
& 1951 & 9 & 8,0 & 3,2 & 1,6 & 3,6 & 1,6 & 1,2 & 88,9 \\
Civitavecchia & 1952 & 10 & 8,0 & 3,1 & 1,6 & 3,5 & 1,7 & 1,2 & 90,7 \\
& 1951 & 6 & 10,9 & 4,2 & 2,4 & 2,6 & 1,4 & 0,9 & 80,0 \\
Napoli & 1952 & 12 & 11,0 & 4,2 & 2,4 & 2,8 & 1,3 & 0,9 & 80,8 \\
& 1953 & 6 & 10,8 & 4,0 & 2,3 & 2,9 & 1,1 & 1,0 & 78,9 \\
Palermo & 1951 & 12 & 11,1 & 4,3 & 2,1 & 2,7 & 1,0 & 0,9 & 27,9 \\
& 1952 & 12 & 11,1 & 4,4 & 2,3 & 2,8 & 0,9 & 1,0 & 30,2 \\
& 1951 & 9 & 11,2 & 4,1 & 1,9 & 3,1 & 1,1 & 1,0 & 17,1 \\
Catania & 1952 & 4 & 11,2 & 4,3 & 2,3 & 3,6 & 1,1 & 1,2 & 14,6 \\
& 1953 & 4 & 11,2 & 4,4 & 2,2 & 2,8 & 1,2 & 0,9 & 7,8 \\
& 1951 & 12 & 6,6 & 3,4 & 1,1 & 1,8 & 1,2 & 0,6 & 33,5 \\
& 1952 & 12 & 6,4 & 3,5 & 1,2 & 1,8 & 1,2 & 0,6 & 28,6
\end{tabular}


Discussione e risultati. - Le taluelle 1 e 2 permettono un confronto immediato fra i valori delle costanti armoniche delle maree e quelli dei corrispondenti livelli medi. Ad un aumento di livello corrisponde un aumento delle costanti armoniche. Particolarmente evidente è la correlazione tra livelli medi e ritardi di fase. La dipendenza risulta più marcata per le componenti più ampie, come per la lunare semidiurna principale M2, la solare semidiurna principale S2 e la lunisolare declinazionale diurna $\mathrm{Kl}$. La relazione appare più incerta per le altre costituenti, ma ciò è giustificato dall'esiguità delle loro ampiezze.

Per determinare anche quantitativamente la dipendenza fra le due grandezze, livello medio e ritardo di fase, sono stati calcolati i rapporti $\Delta g / \Delta$ l. fra la variazione della situazione $g$ e la corrispondente variazione del livello medio $L$. E ciò per ogni componente e per ogni localita. I valori di questi rapporti figurano nella tab. 3. Essi danno pertanto l'aumento (diminuzione) in gradi della situazione $g$ per l'aumento (diminuzione) del livello medio di $1 . \mathrm{cm}$.

TAB. 3. - VALORI DI $\left.\lrcorner_{g}\right\lrcorner l$.

AUMENTO. IN GRADI, DELLA SITUAZIONE $g$ l'ER L'AUMENTO DEI. LIVELLO MEDIO DI $1 \mathrm{~cm}$

\begin{tabular}{|c|c|c|c|c|c|c|}
\hline STAZIONE & M2 & $\mathrm{S} 2$ & $\mathrm{~N} 2$ & K1 & O1 & P1 \\
\hline Tricste & 0,3 & 0,3 & 0,4 & 0,3 & 0,1 & 0,3 \\
\hline Imperia & 0,6 & 0,6 & 2,8 & 1,1 & 2,8 & 1,1 \\
\hline Civitavecchia & 0,6 & $-1,2$ & 2,3 & $-1,1$ & 5,5 & $-1,1$ \\
\hline Napoli & 0,4 & 0,4 & 1,3 & 0,0 & 2,6 & 0,0 \\
\hline Palermo & 0,3 & 0,4 & 0,0 & 0,4 & 1,3 & 0,4 \\
\hline Catania & 0,6 & 1,0 & 1,8 & 0,0 & 0,2 & $(0,0)$ \\
\hline
\end{tabular}

Per Trieste la variazione della situazione è in media di $0^{\circ}, 3$ per $1 \mathrm{~cm}$ di dislivello. I valori dei rapporti corrispondenti alle varie componenti sono in buon accordo fra loro, e ciò dipende dal fatto che sono stati dedotti dal confronto di sei annate. Per il Tirreno i rapporti figurano leggermente più grandi. A Catania si hanno i valori fiù alti, ragrgiungono quasi l'unita, cioè ad $1 \mathrm{~cm}$ di dislivello corrisponde l" di variazione della situazione. Per questi ultimi porti le irregolarità sono più notevoli, ma ciò è dovuto alla incompletezza ed alla insufficienza delle serie mareografiche disponilsili, oltre che alle ampiezze più piccole delle maree. 
T.sB. 4. - VALORI DI $\lrcorner I I\lrcorner l$,

AUMENTO IN $\mathrm{cm}$, DELL'AMPIEZZA $H$

PER L'ALMENTO DEL LIVELLO MEDIO DI $1 \mathrm{~cm}$

\begin{tabular}{|l|r|r|r|r|r|r|}
\hline \multicolumn{1}{|c|}{ STAZIONE } & M2 & S2 & N2 & K1 & OI & P1 \\
\hline Trieste & 0,1 & 0,1 & 0,0 & 0,0 & 0,0 & 0,0 \\
Imperia & 0,0 & $-0,1$ & 0,0 & $-0,1$ & 0,1 & 0,0 \\
Civitavecchia & 0,1 & 0,1 & 0,1 & $-0,2$ & 0,2 & 0.0 \\
Napoli & 0,0 & 0,0 & $-0,1$ & 0,0 & 0,0 & 0,0 \\
Palermo & 0,0 & 0,0 & $-0,1$ & 0,0 & 0,0 & 0,0 \\
Catania & 0,1 & 0,0 & 0,0 & 0,0 & 0,0 & 0,0 \\
& & & & & &
\end{tabular}

Presentiamo pure nella tal. + i ralori dei rapporli $\Delta H / \Delta L$ fra la variazione dell'ampiezza $I I$ dell'onda componente e la corrispon. dente variazione del dislivello. Essi indicano l'aumento in cm dell'ampiezza della componente esaminata per $1 \mathrm{~cm}$ di aumento del livello medio. Data l'esiguiti delle variazioni consilerate $i$ numeri stessi hanno valore solamente informativo. In owni caso, per la componente predominante, risulta che ad un dislivello del mare di $1 \mathrm{~cm}$ corrisponde un aumento dellampiezza dellonda di marea $\mathrm{M} 2 \mathrm{di} 1 \mathrm{~mm}$.

Trieste - Istituto Talassografico - Maggio 1954.

\section{RIASSUNTO}

$\grave{E}$ messo in evidenza la variazione delle costanti armoniche dello. maree di sei stazioni dell'Adriatico, Tirreno e Ionio in dipendenza della contemporanea variazione del medio livello marino. Aumenti o diminuzioni del livello medio di $1 \mathrm{~cm}$ producono aumenti o diminuzioni di auasi $1^{\circ}$ nell angolo della situazione (ritardo di fase) e di circa $1 \mathrm{~mm}$ nell'ampiezza $(\boldsymbol{H})$ delle componenti principali della marea.

\section{SUMMARY}

The variation of the harmonic constants of the tides of six stations of the Adriatic, the Tyrrhenian, and the Ionian seas versus the dependence of the contemporary variations of the mean marine level. 
Increases and decreases of almost $1^{\circ}$ in the place angle (phase retard) and of about $1 \mathrm{~mm}$ in the amplitude (II) of the principal components of the tide have been found.

\section{BIBLIOGRAFIA}

Doonson A. T., Wanıt no: H. D., The Admiralty Tides Tables. Part. III, Instruc, and Tables. Hydrogr. Departm. London 1936.

Por.t. S., Oscillazione annua del Mare Mediterraneo. Arch. di Oceanogr. e Limnol. vol. I, 1, Mem. 288 Comit. Talass. It., 1941.

-, Il graduale aumento del livello del mare a Venezia, Trieste e Poln. Geofisica pura e applicati, IX, 1-2, 1946, Milano.

-, Analisi periodale delle serie dei livelli marini di Trieste e Venezia. Geofisica pura e applicati, $X$ (191.7), 1-2, 29.10, 1 tab., 2 figg.

-, Il graduale aumento del livello del mare lungo le coste italiane. Geofisica pura e applirata, vol. 25, 123-139, 3 tab., Milano, 1953.

--, L'at uale aumento del livello del mare lungo le coste del Mediterraneo. Geofisicat e Meteorologia, vol. 2, 195t, n. 1.2, 13.16, 1 fig.., 2 talb. 